\title{
Association between serum uric acid and proteinuria in patients with type 2 diabetes and stages 1 and 2 chronic kidney disease
}

\author{
Rosita Naseri ${ }^{\mathrm{a}}$, Rahman Amraee ${ }^{\mathrm{b}}$, Anita Eftekharzadeh ${ }^{\mathrm{c}, *}$ \\ ${ }^{a}$ Department of Internal Medicine, School of Medicine, Kermanshah University of Medical Sciences, Kermanshah, Iran \\ ${ }^{\mathrm{b}}$ School of Medicine, Kermanshah University of Medical Sciences, Kermanshah, Iran \\ ${ }^{\mathrm{c}}$ Obesity Research Center, Research Institute for Endocrine Sciences, Shahid Beheshti University of Medical Sciences, Tehran, Iran
}

\section{A R T I C L E I N F O}

\section{Keywords:}

Uric acid

Proteinuria

Diabetic nephropathy

Type 2 diabetes mellitus

\begin{abstract}
A B S T R A C T
Background: - Interaction between serum uric acid level and diabetic nephropathy is a matter of debate. This study is designed to examine the association between serum uric acid (SUA) level and proteinuria in in type 2 diabetes patients with stages 1 and 2 chronic kidney disease (CKD).

Material and methods: - For this cross-sectional study, type 2 diabetic patients aged $\geq 20$ years were recruited from two clinics in Kermanshah city from February 2014 to February 2016. Demographic, anthropometric and metabolic data of participants were obtained. Male and female participants were analyzed separately by logistic regression.

Results: - A total of 432 patients (63.7\% women) with a mean \pm standard deviation (SD) age of $54.68 \pm 9.96$ years were analyzed. Multivariate logistic regression model including age, diabetes duration, body mass index (BMI), estimated glomerular filtration rate (eGFR), fasting blood glucose (FBS), triglyceride (TG), high density lipoprotein cholesterol (HDL-C), low density lipoprotein cholesterol (LDL-C), and SUA showed that in male participants, SUA, FBS, DBP and eGFR significantly predicted proteinuria, while in female gender, SUA, HDL-C, DM duration and age were the independent correlates of proteinuria.

Conclusions: - Serum uric acid significantly predicted proteinuria in both genders. Moreover, in women with type 2 diabetes and stages 1 and 2 CKD, while the association between eGFR and proteinuria was lost, serum uric acid level was independently correlated with the presence of proteinuria.
\end{abstract}

\section{Introduction}

Serum uric acid (SUA) level has been proposed to adversely affect chronic kidney disease (CKD), ${ }^{1}$ however, the causal effect between serum uric acid and renal disease is still debatable. Animal studies revealed that mild hyperuricemia in normal rats lead to hypertension and renal injury. ${ }^{2}$ Also it was shown that lowering uric acid levels in diabetic mice was associated with reduced albuminuria and tubulointerstitial injury. ${ }^{3}$ Moreover, epidemiologic studies showed SUA to be an independent predictor for hypertension. ${ }^{4}$

Notably, studying the relationship between SUA levels and kidney disease is further complicated by the fact that kidney disease per se, affects SUA levels. ${ }^{5}$ A recent study by Yan et al. observed that prevalence of diabetic nephropathy defined as albuminuria $>/=30 \mathrm{mg} /$ $24 \mathrm{~h}$ or estimated glomerular filtration rate $($ GFR) $<90 \mathrm{ml} / \mathrm{min} / 1.73$ $\mathrm{m} 2$ raised with increasing uric acid levels in Chinese population. ${ }^{6}$ Furthermore another investigation on older diabetic patients reported that the independent association of hyperuricemia and albuminuria in diabetic kidney disease was stronger in elderly men. ${ }^{7}$ Likewise, exploring the association between albuminuria and type 1 diabetes mellitus (T1DM), Hovind et al. found that baseline uric acid levels were predictive of persistent macroalbuminuria. ${ }^{8}$ On the other hand, a prospective study results investigating the risk factors for development of non-diabetic chronic kidney disease did not support uric acid as an independent correlates of CKD progression. ${ }^{9}$ Another recent large multi-centric study in type 2 diabetes (T2DM) patients showed that while hyperuricemia was an independent predictor of CKD, in the absence of concomitant metabolic syndrome, there was no association with albuminuria. ${ }^{10}$

In Iran, while varying frequencies for diabetic nephropathy have been reported from different studies, a recent prospective analysis of data from outpatient clinics across the country reported the proportion of diabetic nephropathy as $17.6 \%{ }^{11}$ Noticeably, implementing spot urine tests for detecting albuminuria is still not widely available in

\footnotetext{
* Corresponding author. Research Institute for Endocrine Sciences, No.24 Arabi St, Yemen St, Velenjak, Tehran, Iran.

E-mail address: eftekharzadeh@endocrine.ac.ir (A. Eftekharzadeh).
} 
Table 1

Baseline characteristics.

\begin{tabular}{|c|c|c|c|c|}
\hline Variables & Total & Male & Female & Significance \\
\hline Number (\%) & 432 & $157(36.4)$ & $275(63.4)$ & \\
\hline Age (years) & $54.7(10.0)$ & $56.3(10.5)$ & $53.8(9.5)$ & 0.008 \\
\hline Diabetes duration (months) & $60(36-120)$ & $72(36-120)$ & $60(36-120)$ & 0.368 \\
\hline Smoking (\%) & $43(10.0)$ & $36(23.2)$ & $7(2.6)$ & 0.000 \\
\hline Waist $(\mathrm{cm})$ & $96.0(88.0-102.0)$ & $99.0(94.0-104.0)$ & $94.0(86.0-102.0)$ & 0.000 \\
\hline Abdominal obesity (\%) & $305(72.4)$ & $130(85.0)$ & $175(65.3)$ & 0.000 \\
\hline Body mass index $(\mathrm{kg} / \mathrm{m} 2)$ & $28.8(4.0)$ & $28.6(3.6)$ & $28.9(4.3)$ & 0.080 \\
\hline Obese $(\%)$ & $168(38.9)$ & $57(36.3)$ & $111(40.4)$ & 0.463 \\
\hline Systolic blood pressure (mmHg) & $125(115-141)$ & $130(120-140)$ & $120(110-130)$ & 0.004 \\
\hline Diastolic blood pressure (mmHg) & $75(70-80)$ & $75(70-80)$ & $75(70-80)$ & 0.770 \\
\hline Hemoglobin A1C (\%) & $7.6(6.9-8.8)$ & $7.8(7.0-8.9)$ & $7.6(6.8-8.8)$ & 0.348 \\
\hline eGFR $(\mathrm{ml} / \mathrm{min} / 1.73 \mathrm{~m} 2)$ & $77(69-90)$ & $83(73-93)$ & $74(68-82)$ & 0.000 \\
\hline Fasting blood glucose (mg/dl) & $151.5(114.0-198.0)$ & $150.0(114.0-203.0)$ & $152.5(114.3-194.0)$ & 0.818 \\
\hline Total cholesterol (mg/dl) & $172(148-204)$ & $171(147-198)$ & $175(149-211)$ & 0.276 \\
\hline LDL-C (mg/dl) & $90(73-116)$ & $90(72-113)$ & $90(74-119)$ & 0.325 \\
\hline $\mathrm{HDL}-\mathrm{C}(\mathrm{mg} / \mathrm{dl})$ & $44^{10}$ & $47^{9}$ & $46^{9}$ & 0.000 \\
\hline Triglyceride (mg/dl) & $140(105-198)$ & $140(105-207)$ & 139 (104-197) & 0.397 \\
\hline Uric acid $(\mathrm{mg} / \mathrm{dl})$ & $4.6(3.7-5.7)$ & $5.1(4.0-6.8)$ & $4.3(3.5-5.3)$ & 0.000 \\
\hline Hyperuricemia (\%) & $51(11.8)$ & $35(22.3)$ & $16(5.8)$ & 0.000 \\
\hline Urine Protein (mg/24h) & $93(55-154)$ & $121(63-180)$ & $83(53-135)$ & 0.000 \\
\hline Proteinuria (\%) & $114(26.4)$ & $60(38.2)$ & $54(19.6)$ & 0.000 \\
\hline \multicolumn{5}{|l|}{ Chronic kidney disease } \\
\hline Stage $1(\%)$ & $110(25.5)$ & $60(38.2)$ & $50(18.2)$ & 0.000 \\
\hline Stage $2(\%)$ & $322(74.5)$ & $97(61.8)$ & $225(81.8)$ & \\
\hline
\end{tabular}

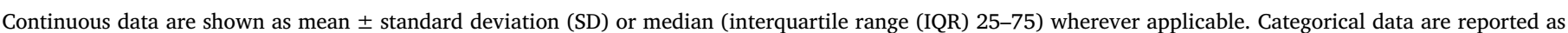

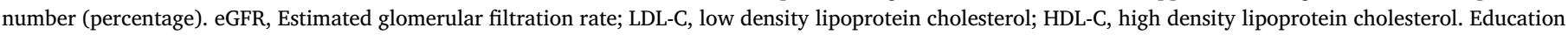
level.

some areas in Iran. However, studies have shown that assessing proteinuria has acceptable cost-effectiveness in detecting early kidney damage in high risk groups. ${ }^{12}$

With respect to above findings, this study aimed to examine the association between serum uric acid levels and proteinuria in type 2 diabetic patients with normal and near normal kidney function (stages 1 and 2 chronic kidney disease) in an urban population of Kermanshah, Iran.

\section{Subjects and methods}

In the present cross-sectional study, patients with type 2 diabetes aged $\geq 20$ years were recruited from endocrinology clinic of Taleghani hospital and Kermanshah University of Medical Sciences clinic from February 2014 to February 2016. To exclude the effect of low GFR on serum uric acid and urine protein, individuals with an eGFR $\leq 60 \mathrm{~mL} /$ $\mathrm{min} / 1.73 \mathrm{~m}^{2}$ as estimated by Modification of Diet in Renal Disease (MDRD) study equation were left out from the study.

The patients' demographic characteristics, including age, sex, education, smoking, duration of diabetes and drug history were recorded during their interview. Weight, height and waist circumference (WC) were measured by trained staff at the same visit. Using digital scales, weight was measured to the nearest $100 \mathrm{~g}$ while participants were minimally clothed, without shoes. Height was measured in a standing position without shoes, using a tape meter. Body mass index (BMI) was calculated as weight in kilograms divided by height in meters squared. WC was measured at the level of the umbilicus using an un-stretched tape meter, without any pressure to the body surface. The reading was recorded to the nearest $0.1 \mathrm{~cm}$. Blood pressure was measured with the participants in the sitting position after $15 \mathrm{~min}$ of rest. A qualified physician took the systolic blood pressure (SBP) and diastolic blood pressure (DBP) twice at a $5 \mathrm{~min}$ interval, after one initial measurement for determining the peak inflation level. Using a standard mercury sphygmomanometer, the mean of the two measurements was considered to be the participant's blood pressure. Laboratory data comprising of serum level of fasting blood sugar (FBS), hemoglobin $\mathrm{A}_{1} \mathrm{C}$ $\left(\mathrm{HbA}_{1} \mathrm{C}\right)$, serum creatinine, serum uric acid, total cholesterol (TC), triglyceride (TG), high-density lipoprotein cholesterol (HDL-C), lowdensity lipoprotein cholesterol (LDL-C), and 24-h urine protein were obtained from the patients' medical records.

Abdominal obesity was described by $\mathrm{WC} \geq 90 \mathrm{~cm}$ in men and women. Proteinuria was defined as excretion of more than $150 \mathrm{mg}$ protein in $24 \mathrm{~h}$ urine. ${ }^{13}$ Hyperuricemia was defined as serum uric acid levels $>7 \mathrm{mg} / \mathrm{dl}$ in men and $>6 \mathrm{mg} / \mathrm{dl}$ in women. Abbreviated MDRD equation was used for estimating the GFR. Chronic kidney disease stages were classified according to the kidney disease outcome quality initiative (K/DOQI) guideline. ${ }^{14}$ Educational level was categorized into 3 groups: illiterate, elementary/middle/high school and university graduates.

Among 455 patients who entered the study, 13 individuals were excluded due to lack of serum uric acid or 24-h urine protein data. Also, another 10 patients with body mass index (BMI) greater than $40 \mathrm{~kg} / \mathrm{m} 2$ or lesser than $18 \mathrm{~kg} / \mathrm{m} 2$ were left out from the study.

All patients signed a written informed consent after receiving full explanation on details and objectives of the study. The study protocol was approved by the Ethic Committee of Kermanshah University of Medical Sciences. Data were collected anonymously and all principles of Helsinki's Declaration were met throughout the study phases.

\subsection{Statistical analysis}

Continuous data were described as mean \pm standard deviation (SD) or median (interquartile range (IQR) 25-75) wherever applicable. Examining the differences between genders were made by $t$-test or Mann-Whitney tests accordingly. Categorical data were reported as number (percentage) and were compared by chi-square test. One way ANOVA and Bonferroni post hoc tests were applied to compare quintiles of serum uric acid levels for Ln transformed urine protein levels. Kolmogorov-Smirnov test was used to assess the normal distribution of data. Univariate and multivariable logistic regression analyses were run to examine the association between serum uric acid levels and selected variables. All analysis were performed by the statistical software SPSS version 22.0. Level of significance was set as $p$ value $<0.05$. Bonferroni correction was used wherever applicable. 
Table 2

Comparison between uric acid quintiles examining differences in 24-h urine protein levels.

\begin{tabular}{|c|c|c|c|c|c|c|c|}
\hline Uric acid quintiles (Q) & Q1 & Q2 & Q3 & Q4 & Q5 & $\mathrm{P}$ value & Post hoc results \\
\hline 24-h Urine protein (male) & 66. $(25-136)$ & $86(52-152)$ & $130(78-198)$ & $141(104-182)$ & $160(107-250)$ & 0.001 & $c, d$ \\
\hline 24-h Urine protein (female) & $75(28-133)$ & $58(37-120)$ & $76(55-115)$ & $88(52-137)$ & $94(67-250)$ & 0.001 & $\mathrm{~d}, \mathrm{~g}, \mathrm{i}$ \\
\hline
\end{tabular}

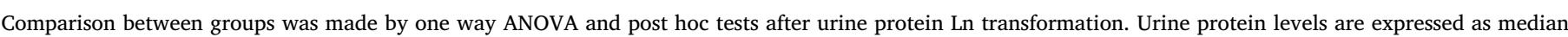

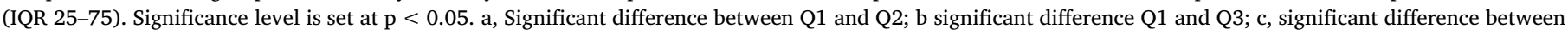

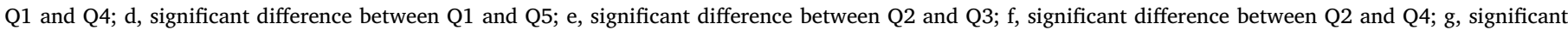

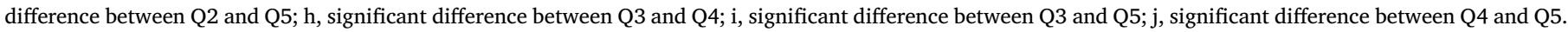

\section{Results}

A total of 432 patients $(63.7 \%$ women) with a mean \pm SD age of $54.7 \pm 10.0$ years were included in our analysis. The mean diabetes duration was 84 months. Fifty one patients (11.5\%) had hyperuricemia and 114 patients $(26.4 \%)$ had proteinuria. Basal characteristics of the included population are depicted in Table 1.

When comparing groups of patients based on quintiles of serum uric acid levels by one way ANOVA test, we observed statistically significant difference in $24 \mathrm{~h}$ urine protein in both genders $(\mathrm{p}=0.001$ ), (Table 2 ).

Univariable logistic regression analysis examining relationship between proteinuria and age, gender, eGFR, SBP, DBP, smoking, HDL-C, total cholesterol, TG, LDL, FBS, uric acid, waist, abdominal obesity, DM duration and BMI showed that there is a significant association between proteinuria and gender, eGFR, HDL-C, uric acid and DM duration (Table 3).

When applying multivariate backward stepwise logistic regression employing a model including age, eGFR, diabetes duration, DBP, FBS, TG, HDL-C, and uric acid, we observed that in male participants, serum uric acid, DBP, eGFR and FBS significantly predicted proteinuria, while in female, serum uric acid, DM duration, HDL-C and age were the significant correlates of proteinuria (Table 4). Notably, the association between eGFR and proteinuria was lost in women.

\section{Discussion}

In the present study, we observed that in patients with type 2 diabetes and stages 1 and 2 chronic kidney disease (normal and near normal kidney function), uric acid level significantly correlated with presence of proteinuria in both gender. However, our results showed no

Table 3

Results of univariable logistic regression examining relationship between selected variables and proteinuria.

\begin{tabular}{lllc}
\hline Variables & Beta & CI 95\% & significance \\
\hline Age & 0.97 & $0.94,1.00$ & 0.064 \\
Gender (male) & 3.67 & $1.81,7.47$ & $<\mathbf{0 . 0 0 1}$ \\
Diabetes duration & 1.01 & $1.00,1.01$ & $\mathbf{0 . 0 1 4}$ \\
Smoking & 1.23 & $0.52,2.87$ & 0.640 \\
Abdominal obesity & 1.36 & $0.47,3.92$ & 0.574 \\
Waist & 1.00 & $0.95,1.05$ & 0.997 \\
Body mass index & 1.06 & $0.95,1.18$ & 0.303 \\
Fasting blood sugar & 1.00 & $1.00,1.01$ & 0.091 \\
Systolic blood pressure & 1.00 & $0.98,1.02$ & 0.996 \\
Diastolic blood pressure & 1.02 & $0.99,1.06$ & 0.189 \\
eGFR & 0.96 & $0.94,0.99$ & $\mathbf{0 . 0 0 2}$ \\
Triglyceride & 1.00 & $1.00,1.00$ & 0.242 \\
Total Cholesterol & 1.00 & $0.99,1.01$ & 0.718 \\
HDL-C & 0.97 & $0.94,1.00$ & $\mathbf{0 . 0 4 7}$ \\
LDL-C & 1.01 & $0.99,1.02$ & 0.278 \\
Uric Acid & 1.35 & $1.10,1.66$ & $\mathbf{0 . 0 0 4}$ \\
Hemoglobin A1C & 1.09 & $0.87,1.36$ & 0.467 \\
\hline
\end{tabular}

Significance level is set at $\mathrm{p}<0.05$. eGFR, estimated glomerular filtration rate; HDL-C, high density lipoprotein cholesterol; LDL-C, low density lipoprotein cholesterol.
Table 4

Multivariate logistic regression analysis (Backward method); predictors of proteinuria.

\begin{tabular}{|c|c|c|c|c|c|}
\hline & $\begin{array}{l}-2 \log \text { likelihood } \\
\text { ratio }\end{array}$ & Variables & Beta & CI 95\% & signific \\
\hline \multirow[t]{4}{*}{ Male } & 159.473 & Uric acid & 1.39 & $1.09,1.78$ & 0.008 \\
\hline & & DBP & 1.05 & $1.01,1.10$ & 0.027 \\
\hline & & $\begin{array}{l}\text { Fasting blood } \\
\text { sugar }\end{array}$ & 1.01 & $1.00,1.02$ & 0.001 \\
\hline & & eGFR & 0.96 & $0.93,0.98$ & 0.002 \\
\hline \multirow[t]{5}{*}{ Female } & 220.366 & Uric acid & 1.32 & $1.03,1.69$ & 0.026 \\
\hline & & $\begin{array}{l}\text { Diabetes } \\
\text { duration }\end{array}$ & 1.01 & $1.00,1.01$ & 0.012 \\
\hline & & eGFR & 0.97 & $0.94,1.00$ & 0.091 \\
\hline & & HDL-C & 0.96 & $0.93,1.00$ & 0.045 \\
\hline & & Age & 0.96 & $0.92,0.99$ & 0.021 \\
\hline
\end{tabular}

Variables examined included age, diabetes duration, eGFR, DBP, FBS, TG, HDL$\mathrm{C}$, and uric acid. eGFR, estimated glomerular filtration rate; DBP, diastolic blood pressure; FBS, fasting blood sugar; HDL-C, high density lipoprotein cholesterol. Significance level is set at $\mathrm{p}<0.05$.

statistically significant relationship between eGFR and proteinuria in women with stages 1 and 2 chronic kidney disease.

To the best of our knowledge this is the first study that has examined the association between serum uric acid level and proteinuria in patients with type 2 diabetes who have stages 1 and 2 chronic kidney disease. Our findings need to be interpreted in the context of our limitations. First, 24-h urine protein test may be imprecise because of its high variation of coefficient as well as errors in urine collection. Nevertheless, this method has been widely employed by some main clinical trials studying renoprotective treatment strategies. ${ }^{15}$ Second, because of the cross-sectional design of the study we cannot draw any conclusion about the causal relationship between serum uric acid level and proteinuria. Third, we did not have data on using Angiotensin II blockers (ARBs), Angiotensin converting enzyme (ACE) inhibitors or Xanthine oxidase inhibitors among our participants. While it might impact the robustness of our results, however, since both genders were affected equally, the results of comparisons made between groups are presumed to remain unaltered.

Our findings about association between uric acid levels and 24-h urine protein are in line with previous studies analyzing the association between albuminuria and SUA in type 2 diabetes. A cross-sectional study by Yan et al. showed that hyperuricemia independently predicted diabetic kidney disease defined as albuminuria $\geq 30 \mathrm{mg} / 24 \mathrm{~h}$ or eGFR $<90 \mathrm{~mL} / \mathrm{min}$ per $1.73 \mathrm{~m} 2{ }^{6}$ Similarly Bonakdaran et al. reported that SUA levels correlated positively with urinary albumin-creatinine ratio. ${ }^{16}$ Another cross-sectional study found that compared to microalbuminuria group, the level of SUA was high in macroalbuminuric diabetic patients. ${ }^{17}$ Likewise, Fukui et al. observed a significantly positive association between SUA and microalbuminuria in men with type 2 DM. $^{18}$ Also, a prospective study in Japan reported that while there was no relationship between SUA and eGFR, low and high SUA levels 
were independent predictors of progression of albuminuria in diabetic patients. ${ }^{19}$ In a longitudinal study, Tsai et al. demonstrated that for each $1 \mathrm{mg} / \mathrm{dl}$ increase in uric acid level from baseline, there was a 7\% increase in the risk of progression toward renal failure. ${ }^{20}$ Zoppini et al. found this number to be as high as $21 \%$ for each SD increment in serum uric acid level. ${ }^{21}$ Another study aiming to ascertain the prognostic value of SUA in diabetic kidney disease progression, found the optimal cut-off values for SUA to be $\leq 6.35 \mathrm{mg} / \mathrm{dl}$ in men and $\leq 5.20 \mathrm{mg} / \mathrm{dl}$ in women, i.e., $10-15 \%$ lower than current cut-offs. ${ }^{22}$ Correspondingly, a small double blinded clinical trial by Momeni et al. observed that low dose allopurinol significantly lowered 24-h urine protein in patients with type $2 \mathrm{DM}$ and proteinuria. ${ }^{23}$

We documented that there was a sex difference in the correlates of proteinuria in our study participants. Sex differences in the epidemiology, progression and prognosis of chronic kidney disease are increasingly acknowledged in the literature, nevertheless, the effect of gender on diabetic nephropathy has remained controversial. ${ }^{24}$ Different methods of estimating GFR and employing non-sex-specific albuminuria cut-offs are among the reasons for these discrepancies. ${ }^{25}$ Interestingly, sexual dimorphism has been reported in the nitric oxide (NO) synthase, chronic inhibition of which has a negative effect on proteinuria. ${ }^{26}$

The multifaceted interactions between serum uric acid level and diabetes micro- and macrovascular complications including diabetic nephropathy have been the subject of a number of studies. ${ }^{27}$ Uric acid which is involved in oxidative stress, is suggested to affect the progression of diabetic nephropathy and might predict the disease. The molecule, produced by the liver, is filtered by glomeruli into the renal tubule. About $90 \%$ of filtered uric acid is reabsorbed by proximal tubule, and approximately $10 \%$ of filtered uric acid is excreted in urine. There are controversies regarding the pathophysiologic role of uric acid in chronic kidney disease. While some studies have shown that uric acid is one of the major antioxidants, others have documented that it can induce oxidative stress in cell. ${ }^{28}$

In conclusion, our study showed that in patients with type 2 diabetes and stages 1 and 2 chronic kidney disease, while eGFR association with proteinuria was lost in women, serum uric acid level showed significant association with proteinuria in both gender. Further studies with prospective design and using sex-specific cut-off for proteinuria are warranted to determine the causal relationship.

\section{Acknowledgments}

The authors would like to thank the clinical Research Development Unit (CRDU) of Emam Reza Hospital, university of Medical sciences, Kermanshah, Iran for their support, cooperation and assistance throughout the period of study.

\section{References}

1. Giordano C, Karasik O, King-Morris K, Asmar A. Uric acid as a marker of kidney disease: review of the current literature. Dis Markers. 2015;2015:6.

2. Sanchez-Lozada LG, Tapia E, Santamaria J, Avila-Casado C, Soto V, Nepomuceno T, et al. Mild hyperuricemia induces vasoconstriction and maintains glomerular hypertension in normal and remnant kidney rats. Kidney Int. 2005;67(1):237-247.

3. Kosugi T, Nakayama T, Heinig M, et al. Effect of lowering uric acid on renal disease in the type 2 diabetic db/db mice. Am J Physiol Renal Physiol. 2009;297(2):F481-F488 Epub 2009/05/22.

4. Feig DI, Mazzali M, Kang DH, et al. Serum uric acid: a risk factor and a target for treatment? J Am Soc Nephrol: JASN (J Am Soc Nephrol). 2006;17(4 Suppl 2):S69-S73 Epub 2006/03/28.

5. Johnson RJ, Kang DH, Feig D, et al. Is there a pathogenetic role for uric acid in hypertension and cardiovascular and renal disease? Hypertension (Dallas, Tex : 1979). 2003;41(6):1183-1190 Epub 2003/04/23.

6. Yan D, Tu Y, Jiang F, et al. Uric acid is independently associated with diabetic kidney disease: a cross-sectional study in a Chinese population. PLoS One. 2015;10(6).

7. Guo M, Niu JY, Li SR, et al. Gender differences in the association between hyperuricemia and diabetic kidney disease in community elderly patients. $J$ Diabetes Complicat. 2015;29(8):1042-1049 Epub 2015/09/22.

8. Hovind P, Rossing P, Tarnow L, Johnson RJ, Parving HH. Serum uric acid as a predictor for development of diabetic nephropathy in type 1 diabetes: an inception cohort study. Diabetes. 2009;58(7):1668-1671 Epub 2009/05/05.

9. Sturm G, Kollerits B, Neyer U, Ritz E, Kronenberg F. Uric acid as a risk factor for progression of non-diabetic chronic kidney disease? The Mild to Moderate Kidney Disease (MMKD) Study. Exp Gerontol. 2008;43(4):347-352 Epub 2008/02/26.

10. Viazzi F, Piscitelli P, Giorda C, et al. Metabolic syndrome, serum uric acid and renal risk in patients with T2D. PLoS One. 2017;12(4):e0176058 Epub 2017/04/20.

11. Esteghamati A, Larijani B, Aghajani MH, et al. Diabetes in Iran: prospective analysis from first nationwide diabetes report of national program for prevention and control of diabetes (NPPCD-2016). Sci Rep. 2017;7(1):13461 Epub 2017/10/19.

12. Boulware LE, Jaar BG, Tarver-Carr ME, Brancati FL, Powe NR. Screening for proteinuria in US adults: a cost-effectiveness analysis. Jama. 2003;290(23):3101-3114 Epub 2003/12/18

13. Carroll MF, Temte JL. Proteinuria in adults: a diagnostic approach. Am Fam Physician. 2000;62(6):1333-1340 Epub 2000/09/30.

14. K/DOQI clinical practice guidelines for chronic kidney disease: evaluation, classification, and stratification. Am J Kidney Dis : The Off J Nat Kidney Found. 2002;39(2 Suppl 1):S1-S266 Epub 2002/03/21.

15. McIntyre NJ, Taal MW. How to measure proteinuria? Curr Opin Nephrol Hypertens. 2008;17(6):600-603 Epub 2008/10/23

16. Bonakdaran S, Hami M, Shakeri MT. Hyperuricemia and albuminuria in patients with type 2 diabetes mellitus. Iranian J Kidney Dis. 2011;5(1):21-24 Epub 2010/12/30

17. Liang CC, Lin PC, Lee MY, et al. Association of serum uric acid concentration with diabetic retinopathy and albuminuria in Taiwanese patients with type 2 diabetes mellitus. Int J Mol Sci. 2016;17(8) Epub 2016/08/05.

18. Fukui M, Tanaka M, Shiraishi E, et al. Serum uric acid is associated with microalbuminuria and subclinical atherosclerosis in men with type 2 diabetes mellitus. Metab Clin Exp. 2008;57(5):625-629 Epub 2008/04/30

19. Hayashino Y, Okamura S, Tsujii S, Ishii H. Association of serum uric acid levels with the risk of development or progression of albuminuria among Japanese patients with type 2 diabetes: a prospective cohort study [Diabetes Distress and Care Registry at Tenri (DDCRT 10)]. Acta Diabetol. 2016;53(4):599-607 Epub 2016/03/05.

20. Tsai CW, Lin SY, Kuo CC, Huang CC. Serum uric acid and progression of kidney disease: a longitudinal analysis and mini-review. PLoS One. 2017;12(1):e0170393 Epub 2017/01/21

21. Zoppini G, Targher G, Chonchol M, et al. Serum uric acid levels and incident chronic kidney disease in patients with type 2 diabetes and preserved kidney function. Diabetes Care. 2012;35(1):99-104 Epub 2011/10/27.

22. Bartakova V, Kuricova K, Pacal L, et al. Hyperuricemia contributes to the faster progression of diabetic kidney disease in type 2 diabetes mellitus. $J$ Diabetes Complicat. 2016;30(7):1300-1307 Epub 2016/06/22.

23. Momeni A, Shahidi S, Seirafian S, Taheri S, Kheiri S. Effect of allopurinol in decreasing proteinuria in type 2 diabetic patients. Iranian journal of kidney diseases. 2010;4(2):128-132 Epub 2010/04/21.

24. Goldberg I, Krause I. The role of gender in chronic kidney disease. European Medical Journal. 2016;1(2):58-64.

25. Yu MK, Katon W, Young BA. Associations between sex and incident chronic kidney disease in a prospective diabetic cohort. Nephrology. 2015;20(7):451-458 Epub 2015/03/27

26. Cobo G, Hecking M, Port FK, et al. Sex and gender differences in chronic kidney disease: progression to end-stage renal disease and haemodialysis. Clinical science (London, England: 1979). 2016;130(14):1147-1163 Epub 2016/06/03.

27. Katsiki N, Papanas N, Fonseca VA, Maltezos E, Mikhailidis DP. Uric acid and diabetes: is there a link? Curr Pharmaceut Des. 2013;19(27):4930-4937 Epub 2013/ $01 / 03$.

28. Jalal DI, Maahs DM, Hovind P, Nakagawa T. Uric acid as a mediator of diabetic nephropathy. Semin Nephrol. 2011;31(5):459-465 Epub 2011/10/18 